\title{
ASYMMETRY AND SKEWNESS IN THE BOTTOM BOUNDARY LAYER : SMALL SCALE EXPERIMENTS AND NUMERICAL MODEL
}

\author{
Céline Berni ${ }^{1,2}$, Leandro Suarez ${ }^{1}$, Hervé Michallet ${ }^{1}$ and Eric Barthélemy ${ }^{1}$
}

\begin{abstract}
This study investigates the non-linearities of wave boundary layers in the surf zone. It mainly focuses on the acceleration skewness or asymmetry. Experiments [e.g. Grasso et al., 2011] show that asymmetry influences the sediment transport. Its influence lies in the fact that asymmetry in velocity (acceleration skewness) tends to transform into velocity skewness within the boundary layer. Analysis by Henderson et al. [2004] predicts a linear relation between $S k_{b} / S k_{\infty}$ and $A s_{\infty} / S k_{\infty}$ where $S k_{b}$ is the dimensionless skewness near the bed, $S k_{\infty}$ the free-stream dimensionless skewness and $A s_{\infty}$ the free-stream dimensionless asymmetry. Numerous experiments were carried out in the LEGI wave flume over a mobile bed composed of lightweight sediments. The quasi-random forcing is a repetition of 2 concatenated bichromatic wave packets. Vertical profiles of velocity are measured in the surf zone. A clear linear relation is shown between these two ratios. The experimental results are compared with the numerical results. A linear relation between skewness and asymmetry is also obtained.
\end{abstract}

Keywords: non linearities, transformation, $k-\omega$ model, mobile bed

\section{INTRODUCTION}

This study investigates the non-linearities of a wave boundary layer in the surf zone. It mainly focuses on the acceleration skewness or asymmetry. Experiments [e.g. Grasso et al., 2011] show that asymmetry influences the sediment transport. Its influence can be interpreted in different ways. Ruessink et al. [2011] listed several physical processes that can explain this influence, out of which asymmetry in velocity (acceleration skewness) tends to transform into velocity skewness within the boundary layer.

This transformation can be explained as follows: an asymmetric wave is an addition of components of different frequencies that are phase-shifted in order to produce steep fronts. If the phase lead of the near-bed velocity is independent of the frequency, the time shift of each component is proportional to its period. Thus, the time shift between components varies when approaching the bed and asymmetry can transform into skewness in the boundary layer.

This simple model explains qualitatively how non linearities transform through the boundary layer. Further analysis by Henderson et al. [2004] quantifies this transformation. Following his arguments, it can be shown, considering a phase lead $\phi$ and a velocity amplitude damping between the near-bed velocity and the free-stream velocity independent of the frequency, that [Berni et al., submitted]:

$$
\frac{S k_{b}}{S k_{\infty}}=\cos (\phi)+\sin (\phi) \frac{A s_{\infty}}{S k_{\infty}}
$$

where $S k_{b}$ is the dimensionless skewness near the bed, $S k_{\infty}$ the free-stream dimensionless skewness and $A s_{\infty}$ the free-stream dimensionless asymmetry.

The purpose of this paper is to study this transformation, both experimentally and numerically.

\section{NUMERICAL MODEL}

The behavior of the wave bottom boundary layer is analyzed using a $k-\omega$ turbulence closure model [Wilcox, 2006 ; Guizien et al., 2003]. The low Reynolds number version of the model is chosen. In this model, the horizontal velocity $u$, the kinetic energy $k$ and the energy dissipation rate $\omega$ are solutions of the following equations :

$$
\begin{aligned}
& \frac{\partial u}{\partial t}=-\frac{1}{\rho} \frac{\partial \bar{P}}{\partial x}+\frac{\partial U}{\partial t}+\frac{\partial}{\partial z}\left(\left(\nu+\nu_{t}\right) \frac{\partial u}{\partial z}\right) \\
& \frac{\partial k}{\partial t}=\nu_{t}\left(\frac{\partial u}{\partial z}\right)^{2}-\beta^{*} k \omega+\frac{\partial}{\partial z}\left(\left(\nu+\sigma_{k} \nu_{t}\right) \frac{\partial k}{\partial z}\right) \\
& \frac{\partial \omega}{\partial t}=\alpha \nu_{t} \frac{\omega}{k}\left(\frac{\partial u}{\partial z}\right)^{2}-\beta \omega^{2}+\frac{\partial}{\partial z}\left(\left(\nu+\sigma_{\omega} \nu_{t}\right) \frac{\partial \omega}{\partial z}\right)
\end{aligned}
$$

where $\partial \bar{P} / \partial x$ is the mean pressure gradient of the flow, $\nu$ the water viscosity, $\nu_{t}$ the turbulent viscosity. The other coefficients and boundary conditions are defined in Guizien et al. [2003]. $x$ is the horizontal direction, $z$ the vertical direction. $U$ is the input velocity of the model.

As we want to study the behaviour of the boundary layer under a non-linear wave, we need to validate the model for such conditions. The results of our model is compared to measurements of Suntoyo et al. [2009] in Utube. The agreement with measurements is good, attesting the capability of the model to reproduce the dynamic of the wave bottom boundary layer under non-linear waves.

\footnotetext{
${ }^{1}$ LEGI UMR 5519, Domaine universitaire, BP 53, 38041 Grenoble cedex 9, France

2 irstea, UR HHLY, Lyon, France
} 

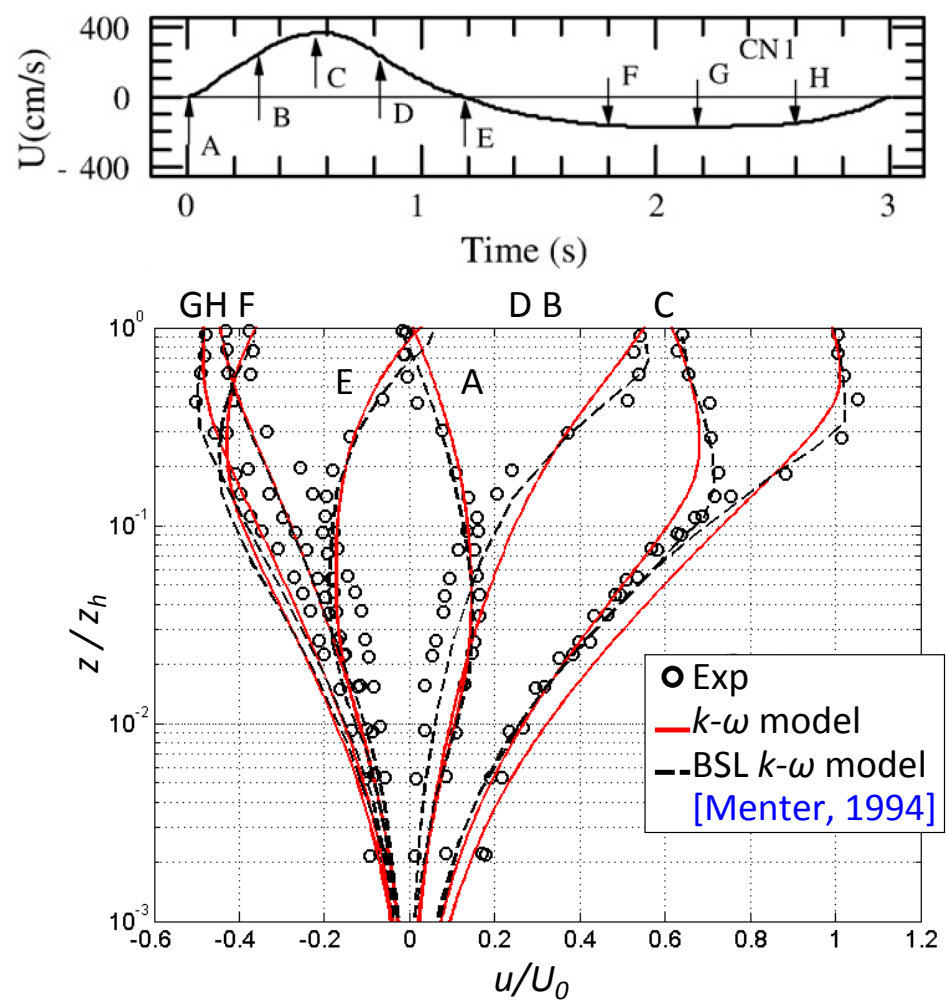

Figure 1: Model validation. Top: time series of the velocity. Bottom: Velocity profiles: comparison between the $k-\omega$ model presented in this article (-), experiments of Suntoyo et al. [2009] (o) and a $k-\omega$ model of Menter [1994] (--).

This model will be used in this study to compute the bottom velocity skewness for different free-stream velocities. The bottom velocity skewness is evaluated at approximately $1 / 10$ of the wave boundary layer thickness over the bed. The free-stream skewness and asymmetry are computed at the maximum value for the orbital velocity. To simulate a wide range of skewness and asymmetries, the formula of Abreu et al. [2010] will be used to compute the input velocity that then will writes:

$$
U(t)=U_{w} f_{r} \frac{\sin (\omega t)+\frac{r \sin \phi}{1+\sqrt{1-r^{2}}}}{1-r \cos (\omega t+\phi)}
$$

where $U_{w}$ is the amplitude of orbital velocity, $\omega=2 \pi / T$ the angular frequency, $r$ the index of skewness ranging from 0 to 0.75 and $\phi$ the waveform parameter ranging from $-\pi / 2$ to 0 . The variable $f_{r}$ is a dimensionless factor, function of $r, f_{r}=\sqrt{1-r^{2}}$, allowing the velocity amplitude to be equal to $U_{w}$.

\section{EXPERIMENTS}

\section{Experimental facility}

The experiments took place in a wave flume of $36 \mathrm{~m}$ long, $55 \mathrm{~cm}$ wide (the LEGI wave flume, see figure 2). The flume is filled with PMMA sediment of low density $\left(1.19 \mathrm{~g} \mathrm{~cm}^{-3}\right)$ forming a beach. The sediment median diameter is $d_{50}=0.64 \mathrm{~mm}$. The corresponding settling velocity is $2.1 \mathrm{~cm} \mathrm{~s}^{-1}$ [see Grasso et al., 2009 , for more details on the experimental facility]. The wave forcing is produced by a computer controlled piston-type wave-maker. There is neither wave absorption nor second-order correction on the wave maker motion.

Capacitive wave gauges are placed in the cross-shore direction of the beach to measure free-surface elevations. Beach profiles are recorded between wave series using an acoustic profiler mounted on a motorized trolley. Crossshore velocity profiles are measured at $X=13 \mathrm{~m}$ of the wave maker with an acoustic Doppler velocity profiler (ADVP) [Hurther, 2001]. The vertical resolution is $3 \mathrm{~mm}$. The sampling frequency for both velocity and freesurface elevation is $50 \mathrm{~Hz}$.

\section{Experimental conditions}

The wave climate is a repetition of a specific wave sequence of duration $T_{s}=53 \mathrm{~s}$. It results of the concatenation of two bichromatic packets with a carrier wave period of $2 \mathrm{~s}$ and $2.5 \mathrm{~s}$ respectively (see figure 3 ). 


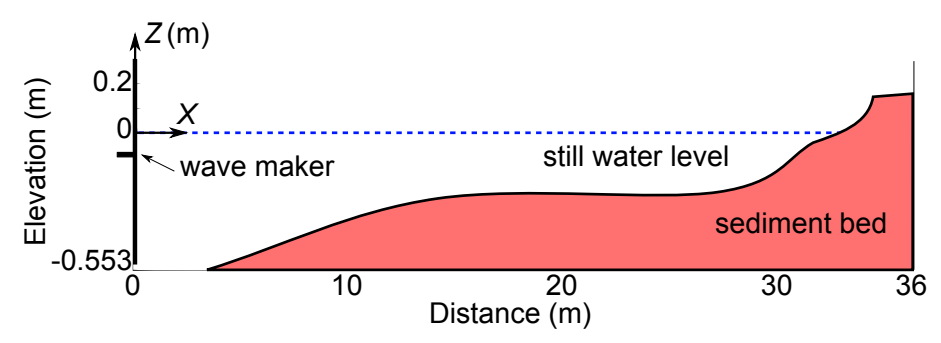

Figure 2: The LEGI wave flume.

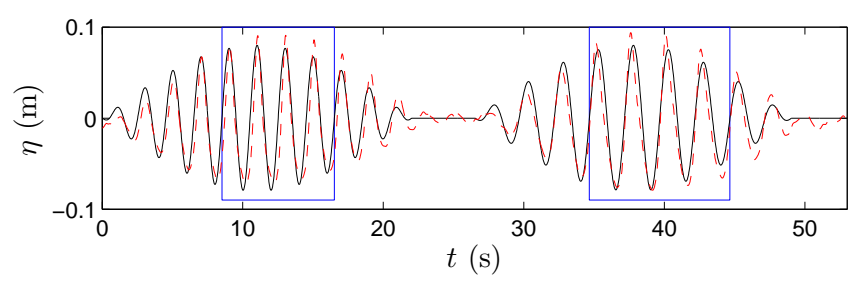

Figure 3: Wave climate: wave maker input data (solid line) and measurements with a capacitive wave gage at $X=2 \mathbf{m}$ (dashed line).

The free-surface elevation $\eta$ for each bichromatic packets writes:

$$
\begin{aligned}
\eta(x, t) & =A \cos \left(\omega_{1} t-k_{1} x\right)+A \cos \left(\omega_{2} t-k_{2} x\right) \\
& =2 A \cos \left(\frac{\omega_{1}-\omega_{2}}{2} t-\frac{k_{1}-k_{2}}{2} x\right) \times \cos \left(\frac{\omega_{1}+\omega_{2}}{2} t-\frac{k_{1}+k_{2}}{2} x\right)
\end{aligned}
$$

where $A=4 \mathrm{~cm}$ for both packets and $x$ is the cross-shore direction. The angular frequencies for the packet with a wave period of $2 \mathrm{~s}$ are $\omega_{1}=3.0 \mathrm{rad} \mathrm{s}^{-1}$ and $\omega_{2}=3.3 \mathrm{rad} \mathrm{s}^{-1}$; for the packet with a wave period of $2.5 \mathrm{~s}$, $\omega_{1}=2.4 \mathrm{rad} \mathrm{s}^{-1}$ and $\omega_{2}=2.7 \mathrm{rad} \mathrm{s}^{-1}$. The wave numbers $k_{1}$ and $k_{2}$ are given by the dispersion relation:

$$
\omega_{i}^{2}=g k_{i} \tanh \left(k_{i} h\right)
$$

where $h$ is the water depth, $h \sim 15 \mathrm{~cm}$ in the experiments presented here. The significant wave height at the wave maker is $16 \mathrm{~cm}$ for both groups.

The initial, intermediate (after 1350 wave sequences of $53 \mathrm{~s}$ ) and final (after 4100 wave sequences) beach profiles are plotted in figure 4. During the experiments a bar progressively formed and migrated onshore. The root mean square wave height $H_{\mathrm{rms}}$ for the initial and final profiles are plotted in the top plot. The break point was overall stationary at a distance of roughly $8 \mathrm{~m}$ from the wave maker. The surf zone extends from there up to the bar trough $(X \sim 20 \mathrm{~m})$. The measurements analyzed in this paper are all recorded within the surf zone at $13 \mathrm{~m}$.

At this specific position, the different experimental parameters can be summarized as follow:

- the bottom Reynolds number $R e_{b}$ ranges between 24000 and 45000 , computed as $R e_{b}=U_{b} h / \nu$ where $\nu$ is the water viscosity and $U_{b}$ is the near-bed velocity measured at the wave crest.

- the orbital amplitude $a$ varies between 5 and $10 \mathrm{~cm}$,

- the Shields number is the order of 0.5 , estimated by

$$
\theta=\frac{1}{2} f_{w} \frac{U^{2}}{g(s-1) d_{50}}
$$

where $U$ is the amplitude of the free-stream velocity $\left(U \sim 0.2 \mathrm{~m} \mathrm{~s}^{-1}\right), s$ is the relative density of the sediment $(s=1.19), d_{50}$ the median diameter and $f_{w}$ is the friction coefficient estimated according to Swart [1974] by:

$$
f_{w}=\exp \left[5.213\left(\frac{2.5 d_{50}}{a}\right)^{0.194}-5.977\right]
$$

- the sheet-flow thickness $\delta_{s}$ is the order of $3 \mathrm{~mm}$, estimated by the Wilson [1987] parameterization

$$
\delta_{s}=10 \theta d_{50}
$$



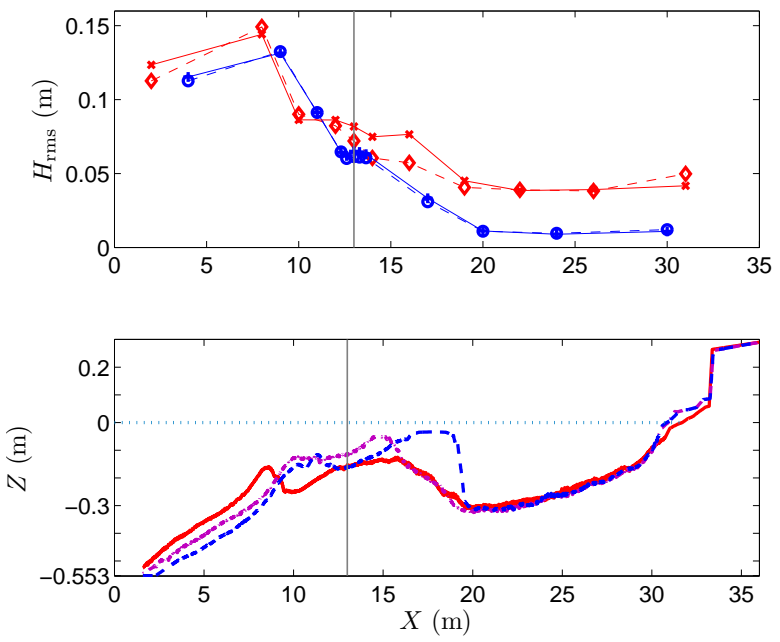

Figure 4: Top: root mean square wave height for the initial $((+): T=2 \mathbf{s} ;(\mathbf{0}): T=2.5 \mathbf{s})$ and the final $((*): T=2 \mathbf{s} ;(\diamond)$ : $T=2.5 \mathbf{s}$ ) topography of the beach. Bottom: initial (bold solid line), intermediate (thin solid line) and final (dashed line) topography of the beach. $X=0$ is the mean position of the wave maker, $Z=0$ is the still free surface (horizontal dotted line). The vertical line indicates the measurement location.
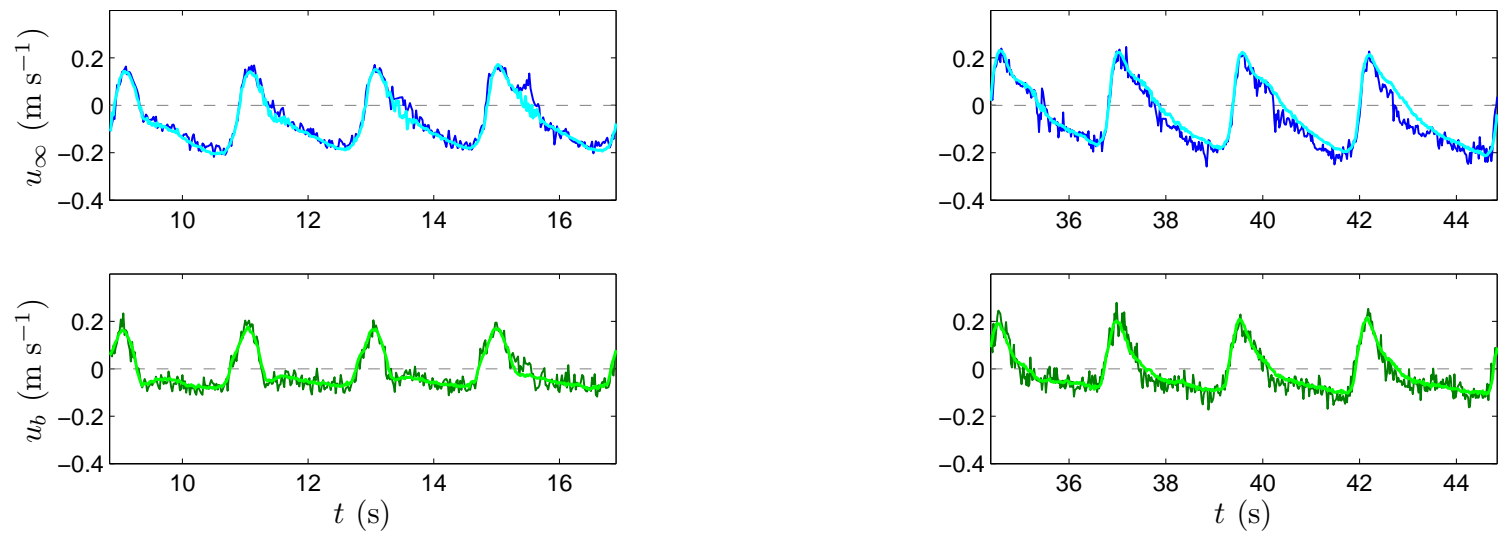

Figure 5: Examples of the time series of the instantaneous free-stream velocity (top) and the instantaneous near-bed velocity (bottom) along with the corresponding ensemble averages (thick light color lines).

- the equivalent roughness length $k_{s}$ is the order of $2 \mathrm{~mm}$, estimated by the Wilson [1989] parameterization

$$
k_{s}=5 \theta d_{50} .
$$

\section{Velocity measurements}

Cross-shore velocity profiles are measured over the bar with an acoustic Doppler velocity profiler (ADVP) [Hurther, 2001]. The vertical resolution is $3 \mathrm{~mm}$. Simultaneously with the velocity profile, the ADVP instrument detects the position of the still bed level [Silva et al., 2009 ; Hurther and Thorne, 2011]. The near-bed velocity $u_{b}$ is defined as the velocity measured in the first sampling volume above the mean position of this still bed level. It is thus measured at an elevation $z_{b}$ between 0 and $3 \mathrm{~mm}$ above the mean still bed level.

Examples of instantaneous velocities at both free-stream and near-bed elevations are given on figure 5. The two groups can be distinguished, the first between $t=1 \mathrm{~s}$ and $t=25 \mathrm{~s}$ the second between $t=25 \mathrm{~s}$ and $t=53 \mathrm{~s}$.

The data presented in this article are provided by ensemble averaging over a set of 30 to 50 sequences in a row. The ensemble average on $N$ realizations is computed as :

$$
\widetilde{u}(t)=\frac{1}{N} \sum_{n=1}^{N} u\left(t+(n-1) T_{s}\right)
$$



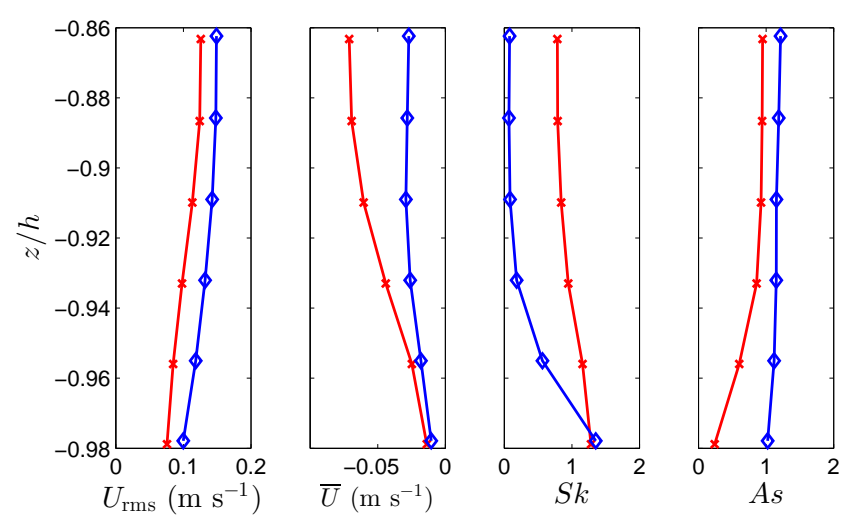

Figure 6: Examples of root-mean-square velocity $U_{\mathrm{rms}}$, mean velocity $\bar{U}$, skewness $S k$ and asymmetry $A s$ profiles computed from ensemble averaged velocity, for the two studied intervals specified in figure 3 corresponding to the two wave forcing: (+): $T=2 \mathbf{s}$; (o): $T=2.5 \mathbf{s}$. The vertical axes is the dimensionless elevation, $Z / h=-1$ stands for the mean position of the bed, $Z / h=0$ for the still free-surface elevation, with the water depth $h=14 \mathrm{~cm}$ at the measuring location.

where $T_{s}$ is the duration of the sequence. For clarity, we simplify the notation by using $u$ for $\widetilde{u}$. During each set of sequences ( $\sim 45$ minutes) the bottom does not evolve significantly in the mean. Within a sequence it-self, the maximum erosion detected is one volume sampling, i.e. $3 \mathrm{~mm}$.

Ensemble averaging reduces the noise and allows reliable velocity measurements. It is superimposed on the instantaneous velocities, on figure 5 , as a thin gray line.

\section{Non linearities}

Time series of the ensemble average of the free-stream and the bottom velocities at the measurement location are shown in figure 5. These data correspond to one representative example amongst the whole set of 41 experiments. The shape of the waves is typical of the surf zone waves, with pinched crests, long troughs, and pitched-forward waves.

The bottom velocity in figure 5 is in contrast highly skewed. Note that the bottom velocity and the free-stream velocity at each crest are almost of the same value while the near-bed troughs velocities are more than $50 \%$ smaller than their counter parts in the free-stream velocities.

In the following analysis of the non-linearities, we chose to only study the four middle waves of each group, identified by a box on the time series in figure 3 . The two time bounds of the box are times with zero-up crossing of the free-stream orbital velocity (without infragravity components). Both intervals are analyzed and studied separately as they correspond to two different periods and therefore to two different forcings. Note that for the example shown on figure 5, asymmetry and skewness are indeed different for both groups. The second group with the $2.5 \mathrm{~s}$ period present a larger asymmetry and a smaller skewness of the free-stream velocity.

For this specific experiment, the vertical profiles of the root-mean-square velocity $U_{\mathrm{rms}}$, the mean velocity $\bar{U}=\langle u\rangle$, the skewness and the asymmetry are plotted in figure 6 for both bichromatic groups. The root mean square value of the velocity, which offers a quantification of the orbital velocity, decreases down to the bed in the wave boundary layer. The mean velocity $\bar{U}$ is negative in the upper part of the profile, distinctive of the presence of an undertow. It decreases deeper down in the boundary layer. The profile surprisingly concaves downwards but it is probably due to the mobile bed. Indeed, O'Donoghue [2004] found similar profiles over a mobile bed. Besides, the measurements of Sparrow et al. [2012] over impermeable and permeable bed also suggest that permeability induces an inflexion in the velocity profiles. Both forcing of different periods show similar behaviour although the free-stream values of the rms and mean velocity are different. In the third panel of the figure is plotted the dimensionless skewness $S k$ of the velocity. It increases down to the bed while the asymmetry decreases as shown in the last panel. The increase of the skewness is larger for the wave period of $2.5 \mathrm{~s}$ that presents a greater freestream asymmetry.

For all the experiments ( 41 groups of at least 30 sequences), dimensionless skewness and asymmetry were computed at the free-stream $(z=\infty)$ and the near-bed $\left(z=z_{b}\right)$ elevations, corresponding to the upper and lower measure presented on figure 6 . The wave boundary layer represents approximately $10 \%$ of the water column and the near-bed elevation is between $1 / 5$ and $1 / 10$ of the wave boundary layer thickness over the bed.

\section{RESULTS}

As the beach shape evolves during the 41 experiments, a large range of values for the free-stream skewness and 

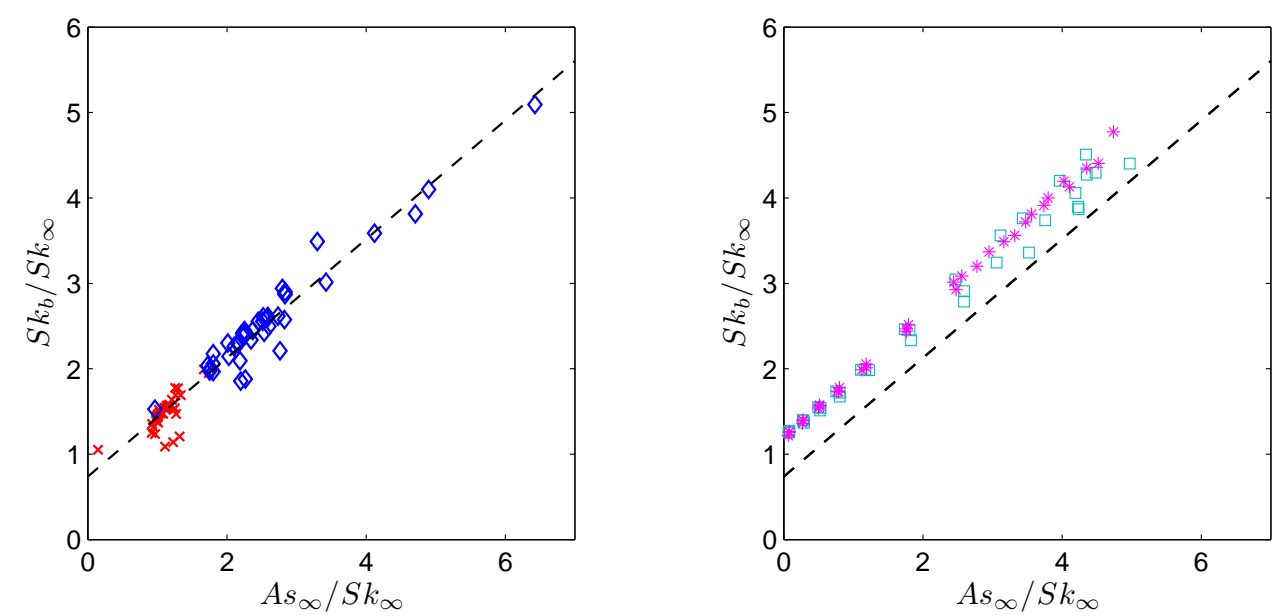

Figure 7: near-bed and free-stream skewness ratio as a function of the ratio between free-stream asymmetry and skewness; left: experimental data ( $\diamond T=2.5 \mathbf{s} ; \mathbf{x} T=2 \mathbf{s}$ ) and right: numerical data $(\square T=2.5 \mathbf{s} ; \star T=2 \mathbf{s}$ ).

large values of the asymmetry are obtained. In our experiments, the waves of period $2.5 \mathrm{~s}$ are more asymmetric and less skewed than the waves of period $2 \mathrm{~s}$.

The ratios appearing in equation (1) are plotted on figure 7. In the experimental case (left), a clear linear relation is shown between these two ratios no matter the forcing. On the right of figure 7 , the results of the numerical model also show a clear linear relation with the same slope. This slope can be interpreted as $\sin (\phi)$ according to the relation (1) and leads to $\phi=44$ degrees. The scatter is similar to the experimental scatter, and larger for the greater period. The y-intercept is quite different between the numerical model and the experiment. Being greater than one in the first case, it can not be interpreted as $\cos (\phi)$ where $\phi$ is the phase lead. Thus, the analytical relation (1) does not hold for turbulent flow such as the one simulated by the model. Indeed, neither the phase lead nor the attenuation at the bed elevation are independent of the frequency. The linear relation yet holds, offering a way to predict bottom velocity skewness.

The process transforming asymmetry to skewness within the boundary layer is numerically and experimentally confirmed and understood. This transformation results in skewed velocities near the bed that lead directly to net sediment transport.

\section{ACKNOWLEDGMENTS}

The technical support of Jean-Marc Barnoud, Muriel Lagauzère and Pierre-Alain Barraud is gratefully acknowledged. The first author's PhD was funded by the French ministry MESR and Grenoble INP, the second author has a PhD Grant of the CONYCIT. This study was partly funded by the project MODLIT (DGASHOM/INSU-RELIEFS) and the project BARCAN (INSU-LEFE).

\section{REFERENCES}

Abreu, T., P. A. Silva, F. Sancho, and A. Temperville (2010). Analytical approximate wave form for asymmetric waves. Coastal Eng., 57(7):656-667.

Berni, C., H. Michallet, and E. Barthélemy (submitted), Surf zone cross-shore boundary layer velocity asymmetry and skewness: an experimental study on a mobile bed, J. Geophys. Res..

Grasso, F., H. Michallet, E. Barthélemy and R. Certain (2009), Physical modeling of intermediate cross-shore beach morphology: Transients and equilibrium states, J. Geophys. Res., 114, C09001, doi:10.1029/2009JC005308.

Grasso, F. , H. Michallet, and E. Barthélemy (2011). Sediment transport associated with morphological beach changes forced by irregular asymmetric, skewed waves. J. Geophys. Res., 116(C3):C03020.

Guizien, K., M. Dohmen-Janssen and G. Vittori (2003), 1DV bottom boundary layer modelling under combined wave and current: suspension ejection events and phase lag effects. J. Geophys. Res., 108(C1):3016.

Henderson, S. M., J. S. Allen, and P. A. Newberger (2004), Nearshore sandbar migration predicted by an eddydiffusive boundary layer model. J. Geophys. Res., 109, C05025, 109.

Hurther, D. (2001), 3-D acoustic Doppler velocimetry and turbulence in open-channel flow, Ph.D. thesis, École Polytechnique Fédérale de Lausanne.

Hurther, D. and P. D. Thorne (2011), Suspension and near-bed load sediment transport processes above a migrating, sand-rippled bed under shoaling waves, J. Geophys. Res., 116, C07001, doi:10.1029/2010JC006774. 
Menter, F. R. (1994), Two-equation eddy-viscosity turbulence models for engineering applications. AIAA Journal, 32 (8), pp 1598-1605.

O'Donoghue, T. and S. Wright (2006), Flow tunnel measurements of velocities and sand flux in oscillatory sheet flow for well-sorted and graded sands, Coast. Eng., 51, 1163-1184.

Ruessink, B. G., H. Michallet, T. Abreu, F. Sancho, D. A. Van der A, J. J. Van der Werf, and P. A. Silva (2011), Observations of velocities, sand concentrations, and fluxes under velocity-asymmetric oscillatory flows. $J$. Geophys. Res., 116(C3):C03004.

Silva, P. A., Abreu T., H. Michallet, D. Hurther, and F. Sancho (2009), Sheet flow layer structure under oscillatory flow, in River, Coastal and Estuarine Morphodynamics, Santa Fe, Argentina, 21-25 Sept 2009.

Sparrow, K., D. Pokrajac, and D. van der A. (2012). The effect of bed permeability on oscillatory boundary layer flow, Proceedings of the International Conference on Coastal Engineering, 723.

Suntoyo, and H. Tanaka (2009), Effects of bed roughness on turbulent boundary layer and net sediment transport under asymmetric waves, Coast. Eng., 56, 960-969.

Swart, D.H. (1974), A schematization of onshore-offshore transport, paper presented at the 14th International Conference on Coastal Engineering, Coastal Eng. Res. Counc., Copenhagen, Denmark.

Wilcox, D. C. (2006), Turbulence modeling for CFD, 3rd edition. DCW Industries, Inc., La Canada CA.

Wilson, K.C. (1989), Mobile-bed friction at high shear stress, J. Hydraul. Res., 1156, 825-830.

Wilson, K.C. (1989), Analysis of Bed-Load Motion at High Shear Stress, J. Hydraul. Eng., 113, 97-0.13. 